\title{
The concept of perceptive empirical design: you can achieve anything with a laser, ingenuity in the design of the impossible
}

\section{Ray Davies}

Ray Davies, "The concept of perceptive empirical design: you can achieve anything with a laser, ingenuity in the design of the impossible," Proc. SPIE 9666, 11th Education and Training in Optics and Photonics Conference, 96661C (5 June 2009); doi: 10.1117/12.2207769

EDIE Event: Eleventh International Topical Meeting on Education and Training in Optics and Photonics, 2009, St. Asaph, United Kingdom 


\section{The Concept of Perceptive Empirical Design You can achieve anything - with a Laser Ingenuity in the Design of the I mpossible Ray Davies

\author{
Photonics Academy at OpTIC
}

Abstract

In devising an effective approach for the Presentation of Information Data to any group of students, it has to be anticipated that the particular Style of Presentation that is chosen for conveying the new Information Data will influence the ensuing level of stimulation of the Response from each student. New Information Data forms the foundations for new Knowledge, but the ideal purpose of any Presentation is to promote the acquisition of Perceptive Knowledge. Any endeavour to promote Perceptive Knowledge involves considerably more cognitive processing procedures than just the initial delivery and reception of new Information Data. It always is necessary to activate the Awareness of a student as to the Usefulness of any Information Data which is being drawn to their attention for the first time. The Style of Presentation needs to bring into sharp Focus specific characteristics in the communication procedures that illustrate the Significance, the Relevance, and the Intrinsic Value of the new Information Data. However, the real importance of new Information Data lies less in the actual content of the Data itself, and much more in the Implications which are consequential to the existence of that Information Data.

This Paper will highlight an approach for the Presentation of Photonics Information Data in such a way as to augment a student's acquisition of Perceptive Knowledge, by the provision of a coincident forum for Photonics Investigations, with the student's own Imagination, Insight, and Ingenuity then being integrated with the Empirical Evidence to arrive at a proposal to create a new Prototype Design Idea. In providing opportunities for a student to create a highly Innovative Prototype Design, such a student's attention is focused on the Usefulness of the original Information Data, challenged by the potential applications of the Information Data within an Investigative environment, and made aware of the impressive outcomes that are the result of Ingenuity that is prompted by a Design encounter. In such a Learning approach, a student is certain to acquire Perceptive Knowledge.

Key Words: $\quad$ Achievement, Design, Data, Empirical, Imagination, Impossible, Information, Ingenuity, Insight, Investigative, Perceptive, Photon, Prototype, Op-Amp, Optoelectronics.

\section{Presentation}

\section{$1 \quad$ The importance of an appropriate Light Sensing System}

When the Photon energy from a source of Light is incident on the surface of an object, complex quantum state attenuations occur between the incident Photons and the orbital electrons of the surface material atoms, with one consequence being that some of that incident Photon energy subsequently is scattered outwards from that surface. Through these surface quantum interactions between the orbital electrons and the Photons, this scattered Photon energy has been encoded in such a way as to carry new Information Data away from that surface.

This scattered Photon Energy, which now has become encoded with new Information Data, carries that Information Data in three distinct categories, namely:-

Category 1 The Intensity and Brightness characteristics of the scattered Light Energy Photons;

Category 2 The Colours and Wavelengths characteristics of the scattered Light Energy Photons;

Category 3 The relative Phase and Emission characteristics of the scattered Light Energy Photons.

To acquire any useful, and valuable, interpretation of this scattered Photon Information Data, an appropriate sensing system needs to be set up to receive this scattered Light Energy Photon Information Data.

When connected to suitable Optoelectronics circuitry, a Photodiode certainly will respond to the Category 1 Information Data. The human eye, and cameras of all types, normally will respond to both Category 1 and to Category 2 Information Data, whereas photographic Holography encompasses the Light Energy Photon Information Data from Category 1, Category 2, as well as Category 3.

In the Prototype Design work of the Photonics Academy at OpTIC, the first emphasis for students is always placed on their construction of a versatile Optoelectronics sensing system, normally incorporating a Photodiode, to detect the Category 1 characteristics of the Light Energy Photons. Since Low Power Laser Beams are normally being considered, Category 2 is not often an essential consideration, and the coherent nature of the Laser Beam Photons provides a useful opportunity for Category 3 characteristics to be merged into Category 1 characteristics, through the effectiveness of the frequently encountered Interference Patterns. Hence, to achieve a really effective outcome from any Prototype Design, a well designed Category 1 sensor is perfectly adequate for students to create. 
The basic Optoelectronics circuit which is used within the Photonics Academy at OpTIC is shown below, for use with a TSL 250 (visible) or TSL 260 (Infrared) series of Photodiodes.

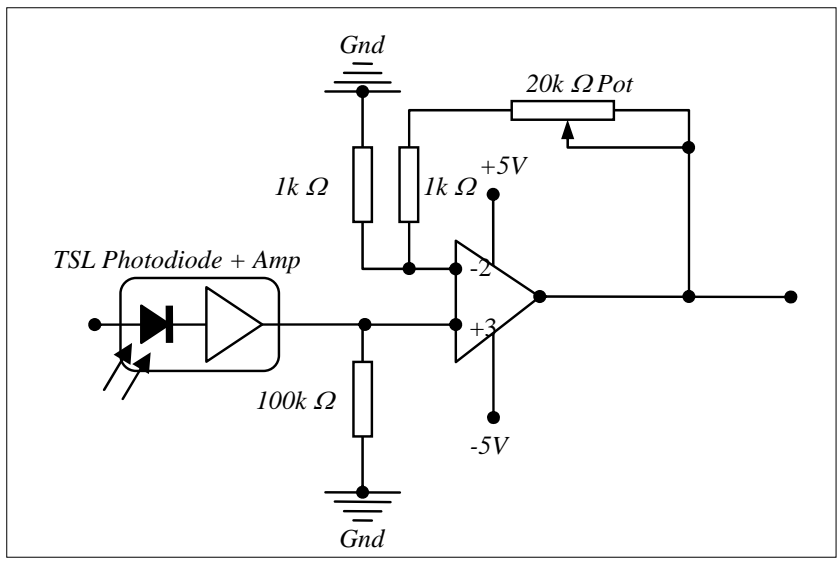

Fig 1 - TLS Photodiode with Additional Variable Gain Operational Amplifier

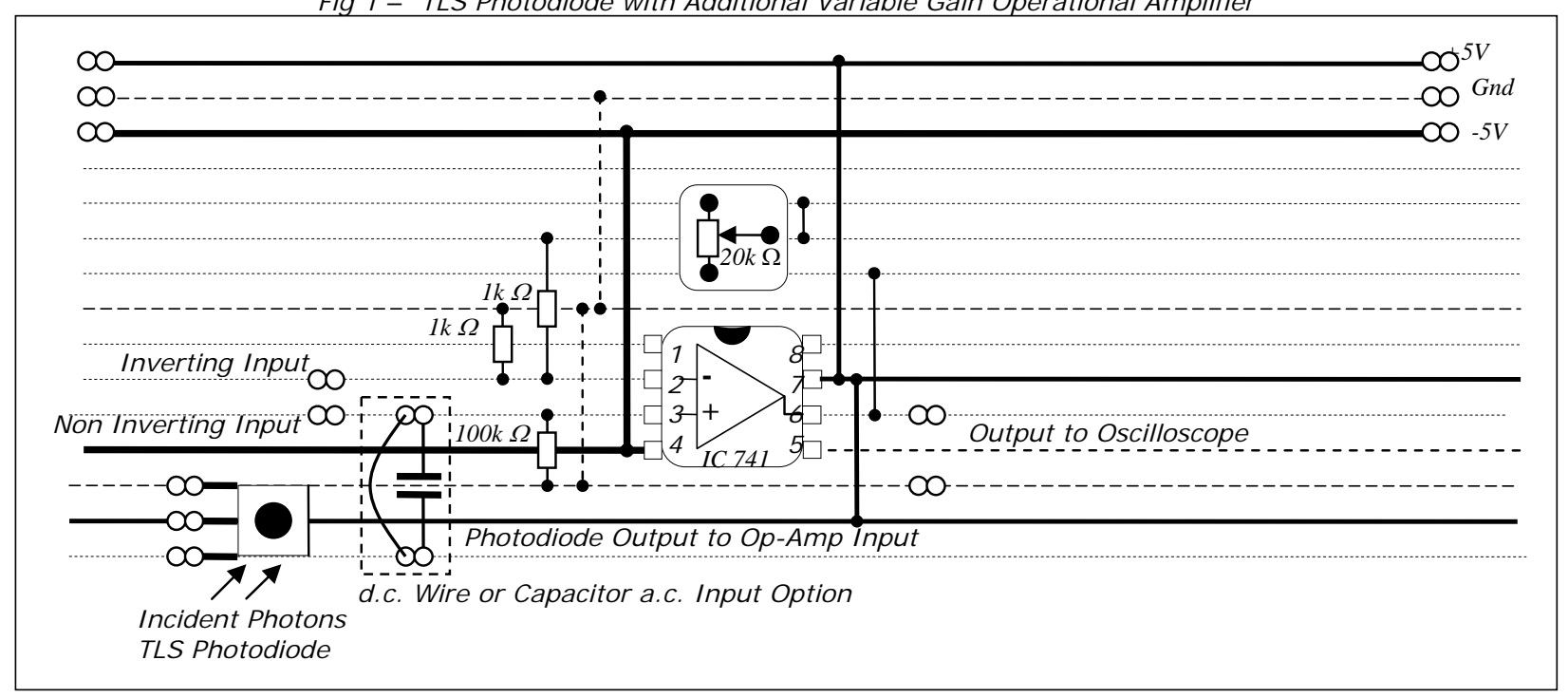

Fig 2 - TLS Photodiode with Strip-board Layout

The above circuit provides a variable Gain Output of up to 20 from the output of the Operational Amplifier. By changing the variable resistor Feedback value used in the above circuit, with the resistance ranging from $5 \mathrm{k} \Omega$ to $1 \mathrm{M} \Omega$, a whole array of bright to even very low levels of Light Intensities can be detected. This circuit is extremely versatile, for it can be used to detect steady, as well as slowly changing, mainly d.c. potential differences, or, if the Pin 3 Input signal is applied through a capacitor, rapidly changing a.c. potential differences can be detected, dependent upon the selection of an appropriately fast response Photodiode.

\section{The Concept of Perceptive Empirical Design}

When the above Category 1 Light Energy Photon Information Data sensor has been constructed, tested, and its full range of versatility investigated by the students of the Photonics Academy at OpTIC, they are then in a position to commence their Perceptive Empirical Design, which eventually always leads to an Innovative Prototype Design outcome.

There are 10 Phases to the Concept of Perceptive Empirical Design, all with a strong focus on student investigations, student observations, and student imagination. Several years of experience in developing the Concept of Perceptive Empirical Design has provided a great deal of evidence to support the recurrent theme that students frequently do reveal imaginative I ngenuity in the Design of the I mpossible. 
4 Flow Diagram for the Concept of Perceptive Empirical Design

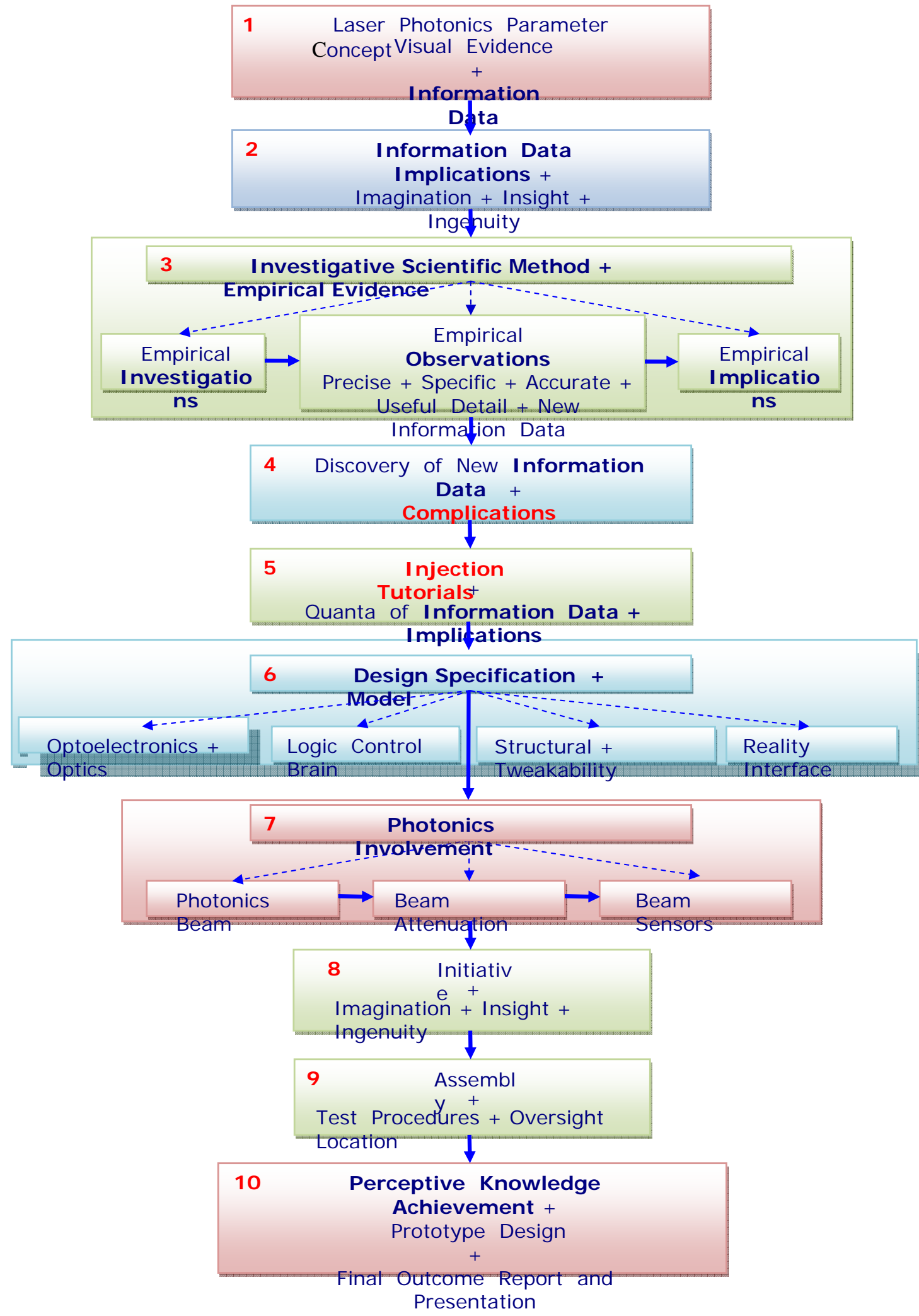

Proc. of SPIE Vol. 9666 96661C-3 
In 1925, Sir J J Thomson (Awarded a Nobel Physics Prize for the Discovery of the Electron) said: -

"The Study of Light has resulted in achievements of

\section{NSI GHT \\ I MAGI NATI ON}

and

I NGENUITY

unsurpassed in any field of mental activity".

If students are encouraged to achieve personal qualities of I nsight, I magination, and I ngenuity, through the medium of Photonics, then any Educational Initiative that places Photonics clearly in the focal plane of any opportunities for students to become personally involved with empirical investigations and observations within Photonics is sure to inspire students towards their own personal desires to acquire perceptive knowledge.

Since 1994, a large number of 14-24 year old students, working with a very similar approach to the Perceptive Empirical Design techniques now used in the Photonics Academy at OpTIC, have designed and constructed well over 200 highly innovative and novel Lower Power Laser Photonics Prototype Design Projects, all of which use Photonics concepts in highly imaginative ways. Many of the students who have been introduced to Perceptive Empirical Design opportunities have been inspired to move towards higher education studies in academic disciplines of Science, Engineering and Medicine, which is evidence of some importance.

Two very frequent responses from Sixth Form Advanced-Level students, who have been introduced to the Teaching technique of Perceptive Empirical Design, have been: -

"Now I know about Photonics, why has no-one ever shown me before that Science is so interesting" and

"Until meeting Photonics, I had completely mapped out my career intentions, but now I have encountered the potential of Photonics, I will have to start again to re-think all of my career planning"

The intention of introducing students to Photonics, through the technique and approach that we have called Perceptive Empirical Design, is so that students are introduced to $21^{\text {st }}$ Century concepts in Light that will enable the students themselves, at a relatively early stage in their scientific development, to change the way in which Light, and Photonics, can be used in highly imaginative ways - which are ways they have designed and created as a direct consequence of their own investigations and perceptive deductions.

Literally within minutes of being introduced to a new Photonics concept, Perceptive Empirical Design students are asked to imagine new ways in which that particular Photonics concept might be used for the benefit of Mankind. This highly focused inspirationally demanding approach to students is in perfect harmony with Pierre Aigrain's definition of Photonics, which he proposed in 1967, namely that:-

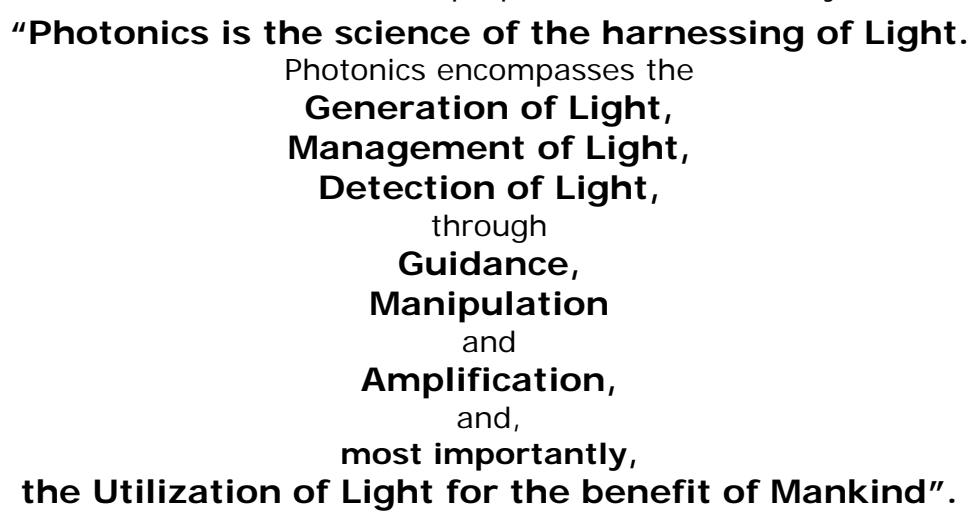

The approach of Perceptive Empirical Design encourages a process of learning by students through their own Investigative Observations, together with the development of new designs that are based upon the implications of the acquisition of their own specific, precise, accurate and useful empirical observations and results.

Perceptive Empirical Design quite naturally leads on to the next phase of the story, namely, the students' personal I ngenuity of the Design of the I mpossible. 
If soldering present a complication for students, the following Prototype Board circuit is recommended.

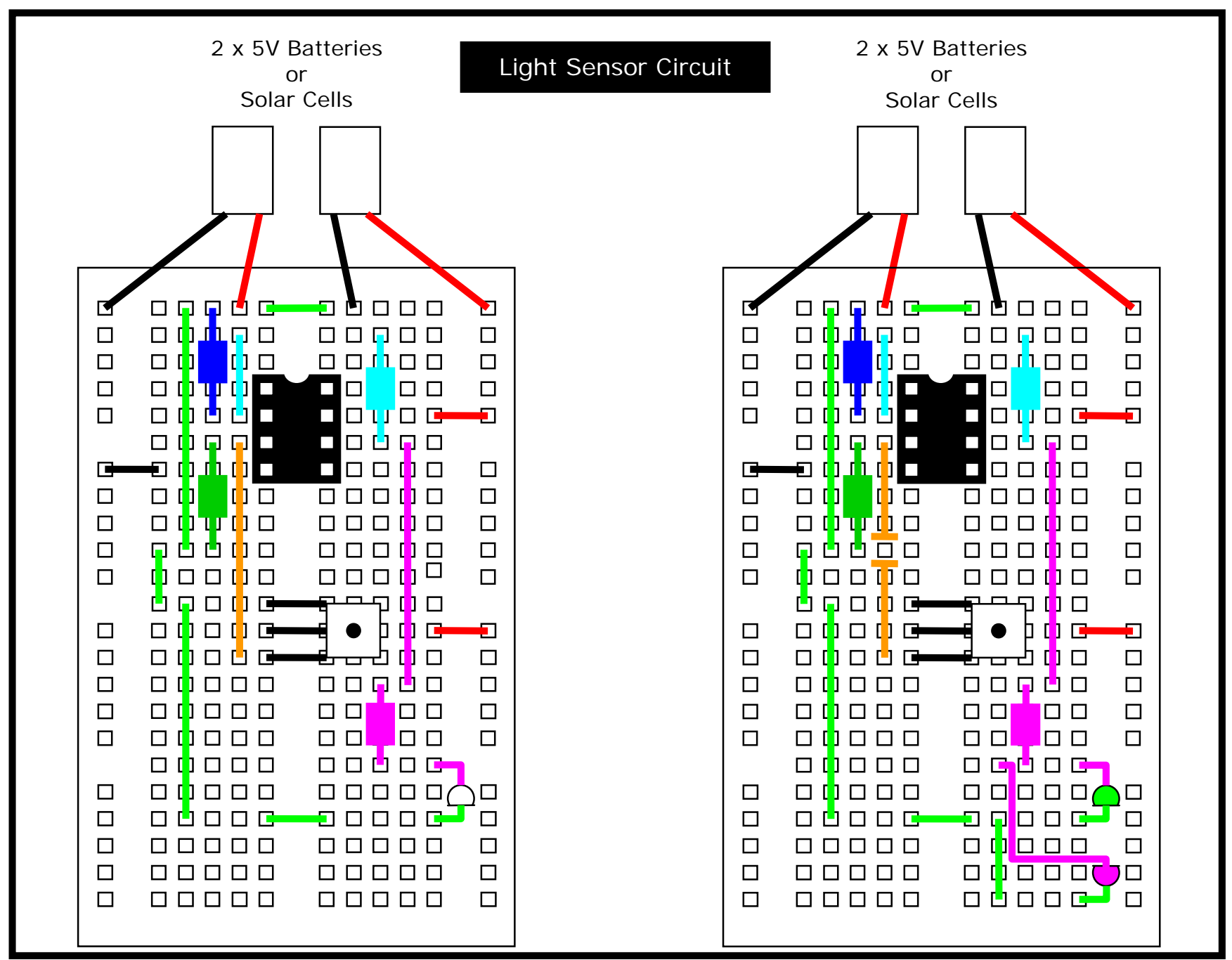

The Left-hand Circuit is a Variable Gain Light Detector, using a Photodiode and Operational Amplifier. This circuit will indicate differing Light Intensity Levels.

The Right-hand Circuit is a Light to Dark / Dark to Light discriminator Light Detector, also using a Photodiode and Operational Amplifier, but with a Capacitor Input to the Operational Amplifier. This circuit discriminates between the Photodiode receiving Light by indicating if the Photodiode receives Light - then Dark, of if the Photodiode receives Dark - then Light. This discriminatory versatility is of immense value in many Photonics applications, particularly in Robotics.

\section{Conclusion}

Perceptive Empirical Design enables students to achieve quite outstanding and impressive changes within Science, through their personal Ingenuity of the Design of the I mpossible.

A student's sense of Personal Achievement in developing an innovative and valuable Prototype, through his, or her, Ingenuity of the Design of the Impossible is of a very high quality factor. 